\title{
Metformin inhibits early stage diethylnitrosamine-induced hepatocarcinogenesis in rats
}

\author{
WOORI JO ${ }^{1,2}$, EUN-SIL YU ${ }^{1,2}$, MINSUN CHANG ${ }^{3}$, HYUN-KYU PARK $^{1,2}$, HYUN-JI CHOI $^{1,2}$, \\ JAE-EUN RYU ${ }^{1,2}$, SUNGWOONG JANG ${ }^{1,2}$, HYO-JU LEE ${ }^{1,2}$, JA-JUNE JANG ${ }^{4}$ and WOO-CHAN SON ${ }^{1,2}$ \\ ${ }^{1}$ Comparative Pathology Core, Asan Institute for Life Sciences; ${ }^{2}$ Department of Pathology, \\ University of Ulsan College of Medicine, Asan Medical Center, Seoul 138-736; \\ ${ }^{3}$ Department of Medical and Pharmaceutical Sciences, Sookmyung Woman's University, \\ Seoul 140-742; ${ }^{4}$ Department of Pathology, Seoul National University College of Medicine, \\ Seoul 110-799, Republic of Korea
}

Received December 1,2014; Accepted September 9, 2015

DOI: $10.3892 / \mathrm{mmr} .2015 .4513$

\begin{abstract}
Antitumor effects of metformin have recently emerged despite its original use for type II diabetes. In the present study, the effects of metformin on the development and recurrence of hepatocellular carcinoma (HCC) were investigated using the diethylnitrosamine (DEN)-induced rat model of HCC. Tumor foci were characterized by gross examination and by histopathological characteristics, including proliferation, hepatic progenitor cell content and the expression of hepatocarcinoma-specific molecular markers. Potential target molecules of metformin were investigated to determine the molecular mechanism underlying the inhibitory effects of metformin on chemically induced liver tumorigenesis. The antitumor effects of metformin were increased by the reduction of surface nodules and decreased the incidence of altered hepatocellular foci, hepatocellular adenoma and carcinoma. Also, decreased expression levels of glutathione S-transferase placental form, proliferating cell nuclear antigen and cytokeratin 8 described the inhibitory effects of metformin on
\end{abstract}

Correspondence to: Professor Woo-Chan Son, Department of Pathology, University of Ulsan College of Medicine, Asan Medical Center, 88 Olympic-ro 43-gil, Songpa-gu, Seoul 138-736, Republic of Korea

E-mail:wcson@amc.seoul.kr

Professor Ja-June Jang, Department of Pathology, Seoul National University College of Medicine, 101 Daehak-ro, Jongno-gu, Seoul 110-799, Republic of Korea

E-mail: tripj@snu.ac.kr

Abbreviations: HCC, hepatocellular carcinoma; DEN diethylnitrosamine; MF, metformin; AHF, altered hepatocellular foci; GST-P, glutathione S-transferase placental form; PCNA, proliferating cell nuclear antigen; CK8, cytokeratin 8; AMPK, AMP-activated protein kinase

Key words: rat, metformin, diethylnitrosamine, hepatocellular carcinoma, AMP-activated protein kinase
HCC. In the present study, Wistar rats receiving treatment with DEN were administered metformin for 16 weeks. In addition, metformin suppressed liver tumorigenesis via an AMPK-dependent pathway. These results suggested that metformin has promising effects on the early stage of HCC in rats. Therefore, metformin may be used for the prevention of HCC recurrence following primary chemotherapy for HCC and/or for high-risk patients, including chronic hepatitis and cirrhosis.

\section{Introduction}

Hepatocellular carcinoma (HCC) is the sixth most frequently diagnosed type of cancer and the third leading cause of cancer mortality (1). In Asia, liver cancer is one of the most common types of cancer (1). The majority of cases are associated with chronic liver disease, including cirrhosis or hepatitis (2). The development of HCC involves sequential morphological changes from hepatic tissue with no recorded tumor complication, to preneoplastic lesions and subsequently to neoplastic lesions $(3,4)$. HCC can be removed through surgical liver resection and liver transplantation. However, the survival rate of patients who have already had surgical resection is $<25 \%$ (5) and liver cancer often reoccurs within 2 years. Furthermore, current chemotherapeutic drugs cannot effectively control tumor progression, and drug resistance is common. Therefore, novel chemotherapeutic agents are urgently required to inhibit $\mathrm{HCC}$ at an early stage, to improve the overall survival rate of patients with HCC.

Several rodent HCC models have been developed to investigate the pathogenesis, treatment and prevention of liver cancer. Chemically-induced hepatocarcinogenesis provides a valuable model for investigating the molecular biology of hepatocarcinogenesis, particularly in its early stages, for various reasons cited previously (6). Diethylnitrosamine (DEN), a DNA alkylating agent, is a widely used to induce carcinogenesis in the rodent model of HCC.

Metformin, a biguanide, is one of the most widely prescribed oral glucose-lowering agents used in the treatment of type II diabetes (7) and has a very low rate of toxicity. 
In addition to its antidiabetic effects, various in vitro and in vivo experiments revealed the anticancer characteristics of metformin (8-11). Previously, other groups revealed that metformin effectively inhibits HCC in mice (12). In addition, the efficiency of metformin in decreasing the incidence of liver cancer has been supported by other in vitro, epidemiological and animal studies $(13,14)$, supporting the potential of its use as an anticancer drug.

In addition, inactivation of AMP-activated protein kinase (AMPK) is associated with tumorigenesis and several malignant cancer types. Zheng et al (15) observed the phosphorylation status of AMPK in HCC and revealed that AMPK acts as a negative regulator in the liver, and loss of the inhibitory effects of AMPK contributes to the progression and invasion of HCC (15). The effect of metformin on HCC cells is predominantly dependent on the activation of AMPK (15). However, whether certain of the antitumor effects of metformin on HCC in vivo are dependent of AMPK remains to be elucidated.

In the present study, the effects of metformin on the development and recurrence of $\mathrm{HCC}$ were investigated using the DEN-induced rat HCC model. To investigate whether metformin affects tumor growth in vivo, a long-term DEN-induced animal model of HCC, causing a high incidence of malignant tumor generation and cirrhosis similarly observed in humans, was used. Concomitant treatment with DEN and metformin was performed to determine whether any metformin-mediated inhibitory effects occurred during the development of HCC.

\section{Materials and methods}

Chemicals and reagents. DEN was purchased from Sigma-Aldrich (St. Louis, MO, USA). Metformin hydrochloride was supplied by Danashmand Organic Private Ltd. (Maharashtra, India), and its purity was determined using standard and high-performance liquid chromatography. Metformin was dissolved in water at a concentration of $0.5 \mathrm{mg} / \mathrm{ml}$ and DEN was dissolved in $0.9 \%$ saline at $50 \mathrm{mg} / \mathrm{kg}$. All antibodies were purchased from Abcam (Cambridge, MA, USA), unless otherwise stated. All molecular biology reagents were molecular biology grade or higher, unless otherwise stated.

Animals, husbandry, and experimental design. A total of 200 male, 6-week-old, Wistar rats, with a mean body weight of $180.75 \pm 5.5 \mathrm{~g}$, were obtained from Orient Bio, Inc. (Yongin, Kyungki, Korea) and were quarantined for 7 days prior to the initiation of the investigation. The rats were maintained at the laboratory animal facility of the Asan Institute for Life Sciences under specific pathogen-free conditions, as indicated by the guidelines of the Animal Care and Use Committee of Asan Institute for Life Sciences.

The rats were housed in environmental conditions with a temperature of $20-24^{\circ} \mathrm{C}$, relative humidity of $50 \pm 10 \%, 12 \mathrm{~h}$ light/dark cycles, illumination at 150-300 Lux and ventilation 10-20 times/hour, which were monitored every hour for $24 \mathrm{~h}$ and maintained within acceptable ranges throughout the present study. The rats were housed three per cage at the beginning of the study and fed an autoclaved pellet diet (Lab
Diet \#5002; PMI Nutrition International LLC, St. Louis, MO, USA) ad libitum.

The study protocol was reviewed and approved by the Institutional Animal Care and Use Committee of Asan Institute for Life Sciences (IACUC no. 2012-01-208). The rats were divided into four groups (60 rats/group in A and $\mathrm{B}$; 40 rats/group in $\mathrm{C}$ and $\mathrm{D})$. All rats in Groups $\mathrm{A}$ and $\mathrm{B}$ received weekly intraperitoneal injections of $50 \mathrm{mg} / \mathrm{kg}$ body weight of DEN dissolved in $0.9 \% \mathrm{NaCl}$ solution (Daehan Pharm Co, Ltd., Kyungki, Korea) over a period of 16 weeks. The rats in groups $\mathrm{B}$ and $\mathrm{C}$ received ad libitum access to $0.5 \mathrm{mg} / \mathrm{ml}$ metformin dissolved in the drinking water. Group D, the negative control group, was provided drinking water ad libitum. All clinical findings were recorded daily, and body weights, food and water intake were measured on a weekly basis. At the end of the study, all animals were weighed and anesthetized with 4\% isoflurane (cat. no. 400-326-09; Baxter, Deerfield, IL, USA). The animals were subsequently sacrificed by exsanguination from the abdominal aorta.

Histopathological and immunohistochemical analysis. On completion of the treatment, the rats were euthanized under isoflurane anesthesia, and their livers were excised and weighed by electronic microbalance (Ohaus Corporation, Pine Brook, NJ, USA). Following a hepatectomy in all rats, the number of surface nodules on the liver was grossly investigated by visual inspection [Miss Woori Jo (University of Ulsan College of Medicine) performed the gross inspection in a blinded manner].

For histopathological and immunohistochemical evaluation, and western blot analysis, the medial, left and right lateral lobes of the liver and the tumor lesions were separated, fresh-frozen at $-196^{\circ} \mathrm{C}$ in liquid nitrogen and stored at $-80^{\circ} \mathrm{C}$ until further analysis. The remainder of the liver tissue was fixed in $10 \%$ neutral buffered formalin (Sigma-Aldrich) for histological evaluation. A tissue processor (Thermo Fisher Scientific, Inc., Runcorn, UK) was used to prepare liver samples from the formalin-fixed samples for analysis by fixing, staining and dehydrating. The paraffin-embedded tissue blocks were cut at a 3- $\mu$ m thickness and mounted onto glass slides. Staining was performed with hematoxylin (YD-Diagnostics, Kyungki, Korea) and eosin (BBC Biochemicals, Mount Vernon, WA, USA) using an autostainer (Leica ST5015; Leica Biosystems Nussloch GmbH, Nussloch, Germany). Immunohistochemistry was performed using an automated slide preparation system, Benchmark XT (Ventana Medical Systems, Inc., Tucson, AZ, USA).Deparaffinization, epitope retrieval and immunostaining were performed, according to the manufacturer's instructions, using cell conditioning solutions and the Benchmark ultraVIEW diaminobenzidine detection system (Ventana Medical Systems, Inc.). Tumor sections were stained with a monoclonal rabbit anti-rat anti-glutathione S-transferase placental form (GST-P) antibody (cat. no. ab138491; 1:2,000 dilution), a monoclonal, rabbit anti-rat anti-proliferating cell nuclear antigen (PCNA) antibody (cat. no. ab92552; 1:1,000) and a polyclonal, rabbit anti-rat anti-cytokeratin 8 (CK8) antibody (cat. no. ab59400; 1:100). Positive signals were amplified using ultraVIEW copper (Ventana Medical Systems, Inc.), and the sections were counterstained with hematoxylin and bluing reagent (Ventana Medical Systems, Inc.). The number and area 
Table I. Incidence of histopathological changes in the livers of Wistar rats.

Treatment duration

\begin{tabular}{|c|c|c|c|c|c|c|c|c|}
\hline \multirow[b]{2}{*}{ Histopathological change } & \multicolumn{2}{|c|}{12 weeks } & \multicolumn{2}{|c|}{14 weeks } & \multicolumn{2}{|c|}{16 weeks } & \multicolumn{2}{|c|}{18 weeks } \\
\hline & DEN & $\mathrm{DEN}+\mathrm{MF}$ & DEN & $\mathrm{DEN}+\mathrm{MF}$ & DEN & $\mathrm{DEN}+\mathrm{MF}$ & DEN & $\mathrm{DEN}+\mathrm{MF}$ \\
\hline Lesions (n) & 15 & 15 & 15 & 15 & 15 & 15 & 12 & 15 \\
\hline Hepatocellular adenoma & 0 & 0 & 2 & 0 & 5 & 2 & 3 & 5 \\
\hline Hepatocellular carcinoma & 0 & 0 & 1 & 0 & 1 & 0 & 6 & 5 \\
\hline Hemangiosarcoma & 0 & 0 & 0 & 0 & 1 & 0 & 0 & 1 \\
\hline AHF & 2.87 & 2.07 & 9.27 & 6.27 & 9.00 & 5.53 & 14.50 & 12.87 \\
\hline Total (except AHF) & 0 & 0 & 0.2 & 0 & 0.47 & 0.13 & 0.75 & 0.73 \\
\hline
\end{tabular}

DEN, diethylnitrosamine; MF, metformin; AHF, altered hepatocellular foci.

of GST-P-positive foci $>0.2 \mathrm{~mm}^{2}$, and the total area of each liver section were measured using a Panoramic 250 Flash II slide scanner (3D-Histech, Ltd., Budapest, Hungary) and Panoramic viewer software 1.15.2 (3D-Histech, Ltd.) to provide values per $\mathrm{cm}^{2}$ of liver tissue. PCNA staining was quantified in five fields of $2 \mathrm{~mm}^{2}$ each in six rats from each group. In addition, staining for the hepatic progenitor cell marker, cytokeratin 8 (CK8), was quantified in an area of $6 \mathrm{~mm}^{2}$ in three rats from each group. The percentage of the area occupied by the positive cells in the total area occupied by all cells was analyzed using i-Solution Lite version 10.6 (IMT i-Solution, Inc., Vancouver, BC, Canada) and the results were expressed as the percentage of positive cell staining.

Western blotting. The liver tissues were homogenized and the samples were incubated on ice with frequent vortexing for $15 \mathrm{~min}$ and centrifuged for $20 \mathrm{~min}$ at $18,000 \mathrm{x} \mathrm{g}$ and $4^{\circ} \mathrm{C}$. The protein content of each sample was quantified using a bicinchoninic acid kit (Pierce, Rockford, IL, USA). Equivalent quantities of protein lysates $(20 \mu \mathrm{g} /$ lane $)$ were separated on $12 \%$ sodium dodecyl sulfate-polyacrylamide gel electrophoresis gels $\left[\mathrm{dH}_{2} \mathrm{O}, 30 \%\right.$ acrylamide mix (Bio-Rad Laboratories, Inc., Hercules, CA, USA), 1.5M Tris pH 8.8 (Bio-Rad Laboratories, Inc.), 10\% SDS (GenDepot, Barker, TX, USA), $10 \%$ ammonium persulfate (Bio-Rad Laboratories, Inc.) and PlusOne TEMED (GE Healthcare Life Sciences, Freiburg, Germany)]. The proteins were transferred onto polyvinylidene membranes (Bio-Rad Laboratories, Inc.) and the blots were incubated with 5\% non-fat dry milk for $1 \mathrm{~h}$ at room temperature, followed by incubation with the appropriate primary antibodies, including monoclonal rabbit anti-rat anti-phospho (p-)AMPK $\alpha$ antibody (Cell Signaling Technology, Inc., Danvers, MA, USA; cat. no. 2535; 1:500), polyclonal rabbit anti-rat anti-AMPK $\alpha$ antibody (cat. no. ab131512; $1: 500$ ) for $20 \mathrm{~h}$ at $4^{\circ} \mathrm{C}$. The blots were washed with $0.1 \%$ Tris-buffered saline, containing $1 \%$ Tween-20, prior to incubation with anti-rabbit $(1: 10,000)$ immunoglobulin $\mathrm{G}$ peroxidase-conjugated secondary antibodies (cat. no. SA002-500; GenDepot), for 1 hour at room temperature. The membranes were developed with chemiluminescent substrates (Thermo Fisher Scientific, Inc.). Hybridization with monoclonal mouse anti-rat anti- $\beta$-actin antibody (cat. no. ab8224; 1:4,000) was used to confirm equal protein loading.

Statistical analysis. Statistical significance was determined using GraphPad Prism 6 (GraphPad Software, San Diego, CA, USA). The data are presented as the mean \pm standard deviation, with the number of samples indicated in the figure legend. The statistical significance of the differences between groups was analyzed by Student's t-test and a two-way analysis of variance. $\mathrm{P}<0.05$ was considered to indicate a statistically significant difference.

\section{Results}

Survivalrate, tumor-free state and body weight of rats following treatment. The experimental design is shown in Fig. 1A, where the rats in groups $\mathrm{B}$ and $\mathrm{C}$ received $0.5 \mathrm{mg} / \mathrm{ml}$ metformin dissolved in drinking water and rats in groups $\mathrm{A}$ and $\mathrm{B}$ were treated with $50 \mathrm{mg} / \mathrm{kg}$ DEN by intraperitoneal injections weekly for 16 weeks. The survival rate, mean body weight and tumor-free state were determined for each of the groups (Fig. 1), A total of three rats in Group A died during the duration of the experiment. The deaths occurred on days 87, 101 and 115. The survival rate is shown in Fig. 1C. Fig. 1D shows the tumor-free state throughout the present study. Additionally, the mean body weight during the duration of the investigation is shown in Fig. 1B. The body weights of the rats in Groups A and B were $17 \%$ lower compared with those in Groups $C$ and D by the $10^{\text {th }}$ week. Groups A and B, which were DEN-treated groups, demonstrated a significant reduction in body weight compared with the control group from the second week. However, no difference was observed between group A and B. The loss of body weight in the DEN-treated groups, $\mathrm{A}$ and $\mathrm{B}$, and the deaths of rats in Group A, were likely due to the general toxic effects of DEN. Since no deaths occurred in Group B, it is possible that metformin protected the rats from the severe toxicity caused by DEN.

Gross examination and histopathological analysis. Fig. 2A shows the gross picture and the number of liver surface nodes observed upon gross examination. The number of liver nodules 
A

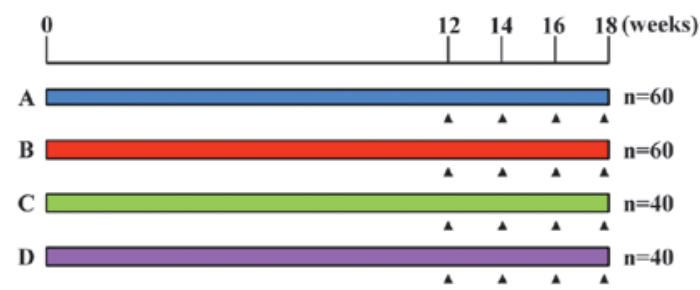

\section{C}

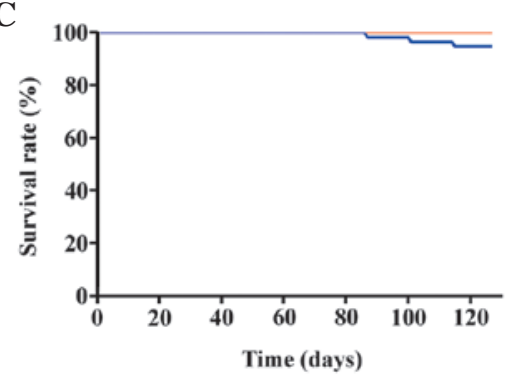

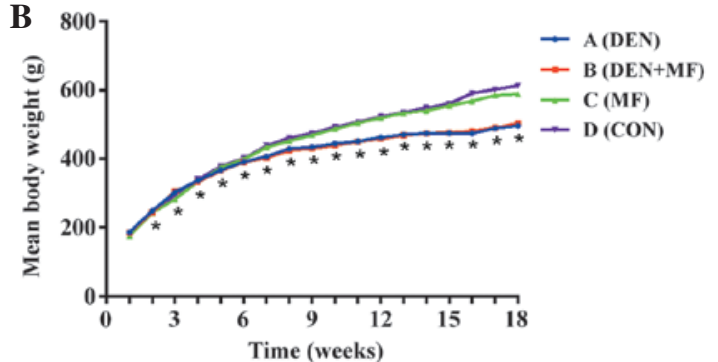

D

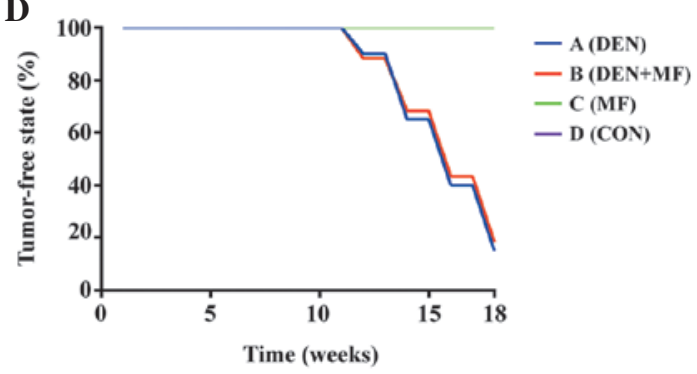

Figure 1. MF successfully inhibits the formation of HCC induced by the long-term administration of DEN. (A) The experimental design placed the samples into four groups: Group A, DEN; Group B, DEN + MF; Group C, MF; Group D, CON. The triangle indicates the time point of sacrifice. (B) The mean body weight $(\mathrm{g})$ of the animals was determined for each group during the experimental duration ("Indicates a significant difference compared with the control rats). (C) The overall survival rate of the animals was determined for the animals used in the present study. (D) The duration of tumor-free state of the animals in each group was determined during the experimental duration. HCC, hepatocellular carcinoma; DEN, diethylnitrosamine; MF, metformin; CON, control.

A

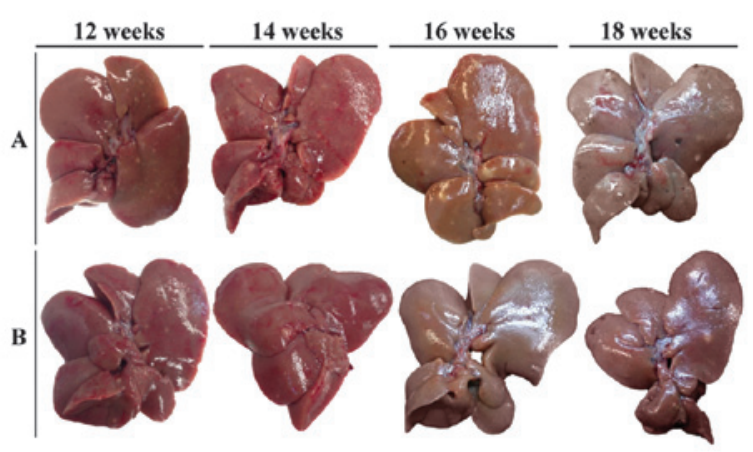

C

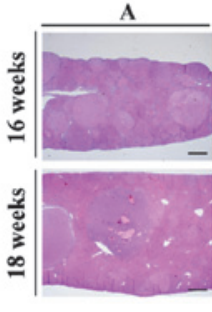

B

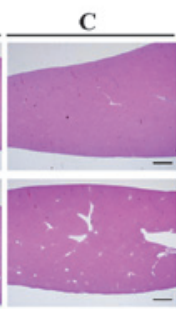

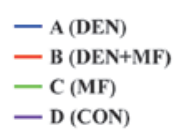

D

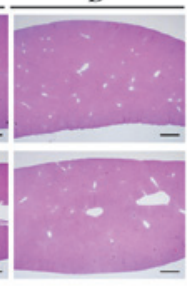

B

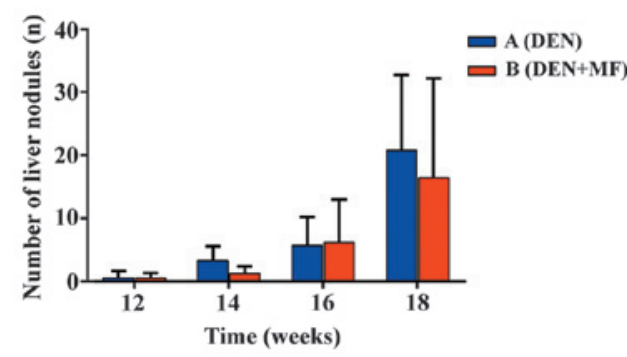

D

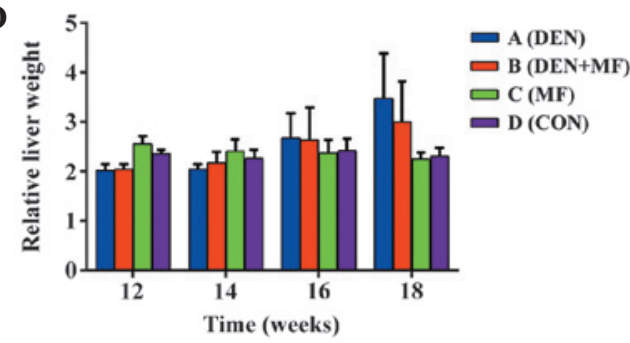

Figure 2. Characterization of DEN-induced HCC and the effects of MF. (A) The gross morphology of representative excised liver from rats treated with DEN alone or DEN in combination of MF. (B) The rats were treated with MF and the number of liver nodules were determined at different time points. Treatment with MF reduced the number of the surface liver nodes. (C) Histopathological sections of the liver were stained with hematoxylin and eosin, and images were captured at weeks 16 and 18 (original magnification, x12.5). (D) The relative liver weights were measured and are expressed as the percentage of the absolute liver weight per total body weight. These were recorded following sacrifice at 12,14, 16 and 18 weeks. HCC, hepatocellular carcinoma; DEN, diethylnitrosamine; MF, metformin; CON, control.

gradually increased over time (Fig. 2B). Representative hematoxylin and eosin micrographs are shown in Fig. 2C. Altered hepatocellular foci (AHF) and tumor nodules were examined in hematoxylin and eosin-stained slides, and were classified as hepatocellular adenoma, HCC and hemangiosarcoma, as shown in Table I. Group B, which was treated with metformin and DEN, exhibited a decreased incidence of AHF, hepatocellular adenoma and HCC compared with Group A, which was treated with DEN alone, between weeks 16 and 18. Cirrhosis was observed in the long-term DEN-treated group.

Absolute and relative liver weights. The final body weights, absolute liver weights and relative liver weights (the relative 
A

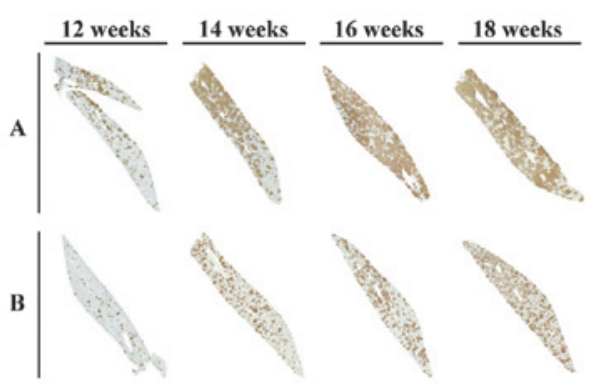

C

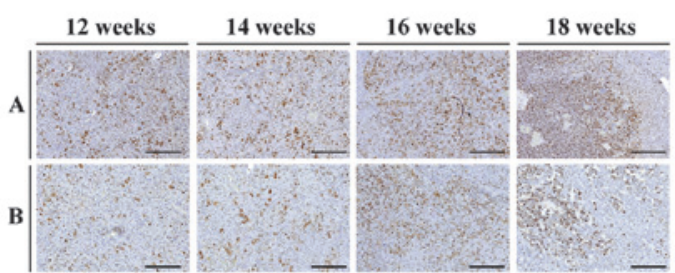

$\mathbf{E}$

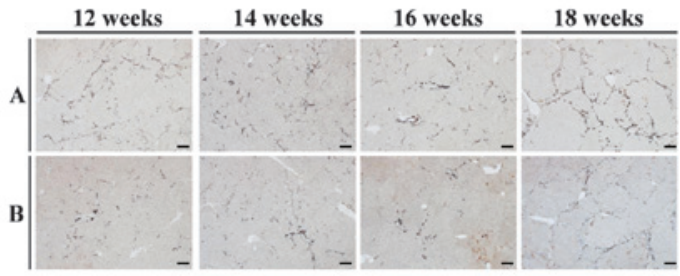

B

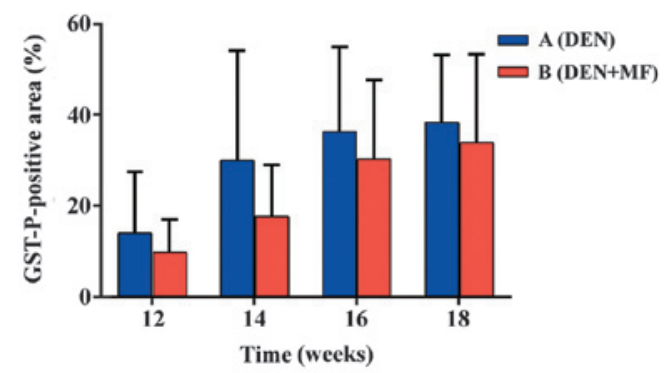

D

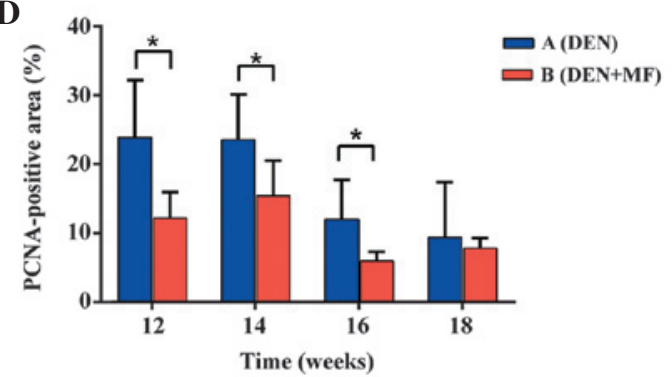

F

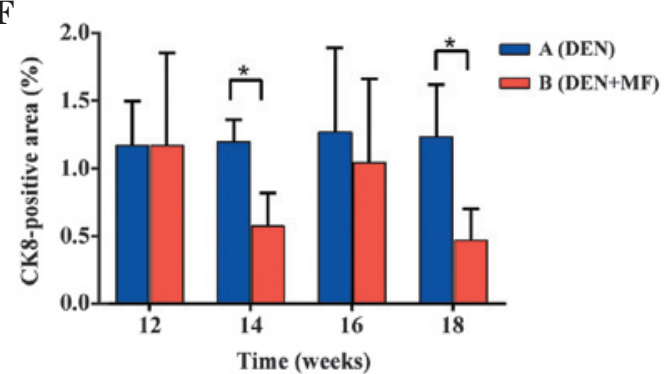

Figure 3. Immunohistochemical analysis of DEN-induced hepatocarcinogenesis and the inhibitory functions of MF. (A) Representative photomicrographs of GST-P antibody-stained rat liver tissues (magnification, x12.5). (B) A decreased GST-P-positive area was observed in the hepatic tissues of rats treated with DEN and metformin compared with the DEN only group. A brown color indicated GST-P nodules as a preneoplastic lesion. (C) Representative photomicrographs of PCNA antibody-stained rat liver tissues (magnification, x40). (D) A decreased number of PCNA-positive cells was observed in the hepatic tissues of rats treated with DEN and MF compared with the DEN only group. (E) Representative photomicrographs of CK8 positive tissues (magnification, $\mathrm{x} 40$ ). (F) A decreased expression of CK8 was observed in the hepatic tissues of rats treated with DEN and MF compared with the DEN only group. DEN, diethylnitrosamine; MF, metformin; CON, control; CK8, cytokeratin 8; PCNA, proliferating cell nuclear antigen; GST-P, glutathione S-transferase placental form. "P<0.05.

liver weights were obtained by dividing the absolute liver weights by the body weights) were recorded following sacrifice at 12, 14, 16 and 18 weeks (Fig. 2D). Necropsies revealed a marked enlargement of the liver in rats treated with long-term DEN (Groups A and B), which was consistent with the increase in liver weight. Treatment with DEN alone (Group A) markedly increased the relative liver weight by $150.1 \%$, whereas co-treatment with DEN and metformin (Group B) resulted in an increase in the relative liver weight of $130.4 \%$ compared with the control group at 18 weeks. These data suggested that the enlargement of the liver caused by DEN was diminished by treatment with metformin.

Quantitative evaluation of GST-P positive cells. The number and area of GST-P-positive cells as end-point markers for hepatocarcinogenesis, particularly in rats as opposed to mice (16), were compared between the DEN-treated rats and the rats co-treated with metformin. As shown in Fig. 3A and B, the GST-P-positive cells in each group steadily increased over time, however, GST-P positivity in Group A remained higher compared with Group B. These results suggested that a reduction in GST-P-positive cells may represent one mechanism underlying the antitumor activity of metformin.
Quantitative evaluation of PCNA-positive cells. Immunohistochemistry for PCNA was performed to determine whether metformin acts by inhibiting proliferation, as previously reported in vitro (17). PCNA-positive cells were quantified in five high-powered fields in different tumors from metformin-treated and untreated rats. Differences in PCNA-positive cells between Groups A and B were observed at weeks $12,14,16$ and $18(\mathrm{P}<0.05)$. As shown in Fig. 3C and $\mathrm{D}$, the difference between Group A and B decreased over time. This may reflect the formation of tumors from AHF.

Quantitative evaluation of CK8-positive cells. Hepatic progenitor cells in the rodent liver samples were assessed by immunohistochemistry for CK8. Hepatic progenitor cells and reactive ductules were reactive for CK8 (18). The ratio of CK8-positive cells between Group A and B were 1.001, 1.775, 1.221 and 2.639 at weeks $12,14,16$ and 18 , respectively $(\mathrm{P}<0.05)$. Metformin inhibited the proliferation of hepatic progenitor cells, resulting in reduced tumor incidence, consistent with other previous in vitro studies (17). Representative micrographs are shown in Fig. 3E, and quantification of CK8 immunostaining for rats in each group is shown in Fig. 3F. 


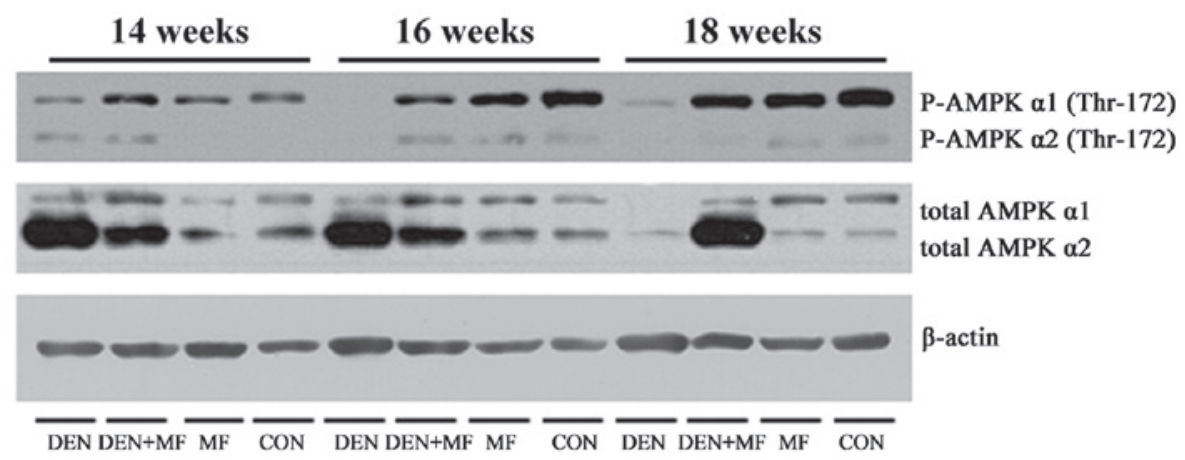

Figure 4. Metformin suppresses liver tumorigenesis via an AMPK-dependent pathway. The expression levels of P-AMPK and total AMPK from six rat livers in each group were analyzed. To determine if MF was important for AMPK activation, the activation status in liver tissues in an HCC model were determined. DEN treatment significantly reduced the level of P-AMPK $\alpha 1$, with marginally increased levels of P-AMPKa1 in groups treated with a combination of DEN and MF, MF alone and CON. DEN, diethylnitrosamine; MF, metformin; CON, control; P-, phosphorylated.

Western blot analysis. Since metformin is important in AMPK activation, the activation status of AMPK was examined in the liver tissues in the HCC model. The total AMPKa and p-AMPK $\alpha$ levels were analyzed in six rat livers in each group. DEN treatment significantly reduced the level of p-AMPK $\alpha 1$, however, the levels of p-AMPK $\alpha 1$ were increased in the co-treatment groups (Fig. 4). These results suggested that the antitumor effect of metformin is, at least partially, dependent on AMPK $\alpha 1$ activation.

\section{Discussion}

In the present study, the number of surface foci, the relative liver weights, the size of GST-P-positive lesions, and the percentage of PCNA- and CK8-positive liver sections were determined to confirm the antitumorigenic effect of metformin on the liver. The data demonstrated that metformin successfully inhibited the occurrence of early-stage hepatocarcinogenesis induced by DEN.

Previous studies performed with animal models treated with DEN have been relatively short-term exposures of $<4$ weeks and rarely induced cirrhosis. Cirrhosis is one of numerous types of liver injury, which can cause HCC. In the present study, DEN was administered up to 16 weeks, which was relatively longer compared with other previous studies. The rats in group A and B received a sufficient exposure to DEN in order to create an animal model, which resembles human HCC. Furthermore, the majority of HCC occurs in livers that are chronically injured as a result of liver cirrhosis or chronic hepatitis. This demonstrates that the initiation of HCC may occur far before HCC can be clinically detected. Certain hepatocarcinogen treatment regimens, including chronic treatment of rats with DEN, also induce AHF, megalocytosis, cholangiofibrosis, cholangiocarcinoma and liver cirrhosis, which are preceded by extensive steatosis, extensive fibrosis and nodular hyperplasia by hepatocytes. Therefore, our long-term DEN treatment model may be a valuable animal model to mimic human HCC tumorigenesis and to assess novel therapies in rats.

The mechanism of action of metformin was investigated in the present study. To assess whether metformin treatment resulted in the activation of AMPK, liver tumors were analyzed for p-AMPK. In the present study, western blot analysis of $\mathrm{HCC}$ tissue revealed a significant decrease in the levels of p-AMPKa1 in the livers of DEN-treated rats, compared with control rats or those treated with metformin, alone or in combination with DEN. Therefore, these results provided clear evidence in support of the AMPK-dependent effects of metformin on HCC.

The inhibitory actions of metformin on tumor growth, including cellular studies, are possibly explained by several mechanisms (19-22). Early previous studies have suggested that metformin may inhibit tumor growth by activating AMPK, an energy sensor $(19,23,24)$. Other studies have demonstrated AMPK-independent effects of metformin on glucose homeostasis and tumor growth in vitro and in vivo $(25,26)$. These studies still contradict certain other studies, including the present study, which demonstrated AMPK activation in the liver by metformin. One possible explanation for the discrepancies between these studies may be the extended duration of treatment compared with other previous studies, which used a short-term treatment (27). In addition, Memmott et al (22) have recently demonstrated that AMPK activation by metformin in the liver may depend on the route of administration and that intraperitoneal, however, not oral, metformin treatment increased the phosphorylation of AMPK in the liver. Intraperitoneal administration is known to result in a higher systemic concentration of metformin compared with oral administration (22). However, oral administration is currently being used in clinical trials, and therefore, the present study selected oral administration for investigation. The present study revealed that phosphorylation of AMPK was increased by oral administration, suggesting that metformin inhibited tumor growth via an AMPK-dependent pathway in the liver when administered orally.

This is the first study, to the best of our knowledge, to demonstrate the antitumor effects of metformin on long-term DEN-treated rat livers. The long-term DEN-treated rat model of HCC is a good model reflecting the status of human HCC and the present study has investigated the inhibitory effects of metformin on HCC. As in a previous study using AMPK as a biomarker for HCC (15), the therapeutic effects of metformin were assessed in a patient-derived xenograft animal model using p-AMPK as a biomarker for HCC in the stage of drug development. These studies may offer an improved insight into our understanding of the effects of metformin on the 
early stage of HCC development in rats and may allow for the appropriate application of metformin for the prevention of $\mathrm{HCC}$ recurrence following treatment of primary HCC. Also, the present study provided a clear rationale for the use of metformin for the cure for the high-risk patients.

\section{Acknowledgements}

The present study was supported by grants from the Korean Health Technology Research and Development Project, Ministry of Health and Welfare, Korea (nos. HI06C0868 and HI10C2014).

\section{References}

1. Jemal A, Center MM, DeSantis C and Ward EM: Global patterns of cancer incidence and mortality rates and trends. Cancer Epidemiol Biomarkers Prev 19: 1893-1907, 2010.

2. Bosch FX, Ribes J, Cléries R and Díaz M: Epidemiology of hepatocellular carcinoma. Clin Liver Dis 9: 191-211, 2005.

3. Maronpot RR, Montgomery CA Jr, Boorman GA and McConnell EE: National toxicology program nomenclature for hepatoproliferative lesions of rats. Toxicol Pathol 14: 263-273, 1986.

4. Eustis SL: The sequential development of cancer: A morphological perspective. Toxicol Lett 49: 267-281, 1989.

5. Wild CP and Hall AJ: Primary prevention of hepatocellular carcinoma in developing countries. Mutat Res 462: 381-393, 2000.

6. Ogawa K: Molecular pathology of early stage chemically induced hepatocarcinogenesis. Pathol Int 59: 605-622, 2009.

7. Kirpichnikov D, McFarlane SI and Sowers JR: Metformin: An update. Ann Intern Med 137: 25-33, 2002.

8. Hirsch HA, Iliopoulos D, Tsichlis PN and Struhl K: Metformin selectively targets cancer stem cells and acts together with chemotherapy to block tumor growth and prolong remission. Cancer Res 69: 7507-7511, 2009.

9. Aljada A and Mousa SA: Metformin and neoplasia: Implications and indications. Pharmacol Ther 133: 108-115, 2012.

10. Decensi A, Puntoni M, Goodwin P, Cazzaniga M, Gennari A, Bonanni B and Gandini S: Metformin and cancer risk in diabetic patients: A systematic review and meta-analysis. Cancer Prev Res (Phila) 3: 1451-1461, 2010.

11. Algire C, Zakikhani M, Blouin MJ, Shuai JH and Pollak M: Metformin attenuates the stimulatory effect of a high-energy diet on in vivo LLC1 carcinoma growth. Endocr Relat Cancer 15 833-839, 2008

12. Bhalla K, Hwang BJ, Dewi RE, Twaddel W, Goloubeva OG, Wong KK, Saxena NK, Biswal S and Girnun GD: Metformin prevents liver tumorigenesis by inhibiting pathways driving hepatic lipogenesis. Cancer Prev Res (Phila) 5: 544-552, 2012.
13. Qu Z, Zhang Y, Liao M, Chen Y, Zhao J and Pan Y: In vitro and in vivo antitumoral action of metformin on hepatocellular carcinoma. Hepatol Res 42: 922-933, 2012.

14. Chen HP, Shieh JJ, Chang CC, Chen TT, Lin JT, Wu MS, Lin JH and Wu CY: Metformin decreases hepatocellular carcinoma risk in a dose-dependent manner: Population-based and in vitro studies. Gut 62: 606-615, 2013.

15. Zheng L, Yang W, Wu F, Wang C, Yu L, Tang L, Qiu B, Li Y, Guo L, Wu M, et al: Prognostic significance of AMPK activation and therapeutic effects of metformin in hepatocellular carcinoma. Clin Cancer Res 19: 5372-5380, 2013.

16. Morimura S, Suzuki T, Hochi S, Yuki A, Nomura K, Kitagawa T, Nagatsu I, Imagawa M and Muramatsu M: Trans-activation of glutathione transferase $\mathrm{P}$ gene during chemical hepatocarcinogenesis of the rat. Proc Natl Acad Sci USA 90: 2065-2068, 1993.

17. Saito T, Chiba T, Yuki K, Zen Y, Oshima M, Koide S, Motoyama T, Ogasawara S, Suzuki E, Ooka Y, et al: Metformin, a diabetes drug, eliminates tumor-initiating hepatocellular carcinoma cells. PLoS One 8: e70010, 2013.

18. Lowes KN, Croager EJ, Olynyk JK, Abraham LJ and Yeoh GC: Oval cell-mediated liver regeneration: Role of cytokines and growth factors. J Gastroenterol Hepatol 18: 4-12, 2003.

19. Zakikhani M, Dowling R, Fantus IG, Sonenberg N and Pollak M: Metformin is an AMP kinase-dependent growth inhibitor for breast cancer cells. Cancer Res 66: 10269-10273, 2006.

20. Rocha GZ, Dias MM, Ropelle ER, Osório-Costa F, Rossato FA, Vercesi AE, Saad MJ and Carvalheira JB: Metformin amplifies chemotherapy-induced AMPK activation and antitumoral growth. Clin Cancer Res 17: 3993-4005, 2011.

21. Kourelis TV and Siegel RD: Metformin and cancer: New applications for an old drug. Med Oncol 29: 1314-1327, 2012.

22. Memmott RM and Dennis PA: LKB1 and mammalian target of rapamycin as predictive factors for the anticancer efficacy of metformin. J Clin Oncol 27: e226; author reply e227, 2009.

23. Zhou G, Myers R, Li Y, Chen Y, Shen X, Fenyk-Melody J, Wu M, Ventre J, Doebber T, Fujii N, et al: Role of AMP-activated protein kinase in mechanism of metformin action. J Clin Invest 108: 1167-1174, 2001.

24. Zakikhani M, Dowling RJ, Sonenberg N and Pollak MN: The effects of adiponectin and metformin on prostate and colon neoplasia involve activation of AMP-activated protein kinase. Cancer Prev Res (Phila) 1: 369-375, 2008.

25. Foretz M, Hébrard S, Leclerc J, Zarrinpashneh E, Soty M, Mithieux G, Sakamoto K, Andreelli F and Viollet B: Metformin inhibits hepatic gluconeogenesis in mice independently of the LKB1/AMPK pathway via a decrease in hepatic energy state. J Clin Invest 120: 2355-2369, 2010.

26. Kalender A, Selvaraj A, Kim SY, Gulati P, Brûlé S, Viollet B, Kemp BE, Bardeesy N, Dennis P, Schlager JJ, et al: Metformin, independent of AMPK, inhibits mTORC1 in a rag GTPase-dependent manner. Cell Metab 11: 390-401, 2010.

27. Shaw RJ, Lamia KA, Vasquez D, Koo SH, Bardeesy N, Depinho RA, Montminy M and Cantley LC: The kinase LKB1 mediates glucose homeostasis in liver and therapeutic effects of metformin. Science 310: 1642-1646, 2005. 